\title{
Tin-induced crystallization of amorphous silicon assisted by a pulsed laser irradiation
}

\author{
V.B. Neimash ${ }^{1}$, V.V. Melnyk ${ }^{1}$, L.L. Fedorenko ${ }^{2}$, P.Ye. Shepeliavyi ${ }^{2, a}$, V.V. Strelchuk ${ }^{2}$, A.S. Nikolayenko ${ }^{2}$, \\ M.V. Isaiev ${ }^{3}$, A.G. Kuzmich ${ }^{3}$ \\ ${ }^{1}$ Institute of Physics, National Academy of Sciences of Ukraine, \\ 46, prospect Nauky, 03680 Kyiv, Ukraine \\ ${ }^{2}$ Institute of Semiconductor Physics, National Academy of Sciences of Ukraine, \\ 45, prospect Nauky, 03680 Kyiv, Ukraine \\ ${ }^{3}$ Taras Shevchenko National University of Kyiv, Faculty of Physics, \\ 60, Volodymyrska str., 01601 Kyiv, Ukraine \\ a) Author to whom correspondence should be addressed. \\ Electronic addresses: pshepeliavyi@gmail.com
}

\begin{abstract}
The process of tin-induced crystallization of amorphous silicon under the influence of different types of laser irradiation was investigated using the method of Raman scattering by thin-film Si-Sn-Si structures. The dependences of the size and concentration of $\mathrm{Si}$ nanocrystals on the power of laser radiation was experimentally evaluated and analyzed. As sources of excitation pulse laser radiation with the pulses duration equal to $20 \mathrm{~ns}$ and $150 \mu$ s and wavelengths equal to 535 and $1070 \mathrm{~nm}$ was used. The possibility of efficient tin-induced transformation of silicon from amorphous phase to crystalline one in the 200-nm thick layers of a-Si under the action of laser pulses with duration equal to $20 \mathrm{~ns}$ was shown. The spatial and temporal distributions of laser induced temperature rise was calculated to interpret experimental results.
\end{abstract}

Keywords: thin film, metal-induced crystallization, pulsed laser treatment.

Manuscript received 02.10.17; revised version received 06.11.17; accepted for publication 07.12.17; published online 07.12.17.

\section{Introduction}

The film composite "Si nanocrystals in the matrix of amorphous $\mathrm{Si}$ " is promising material for the next generation of solar cells (SC) based on quantum dots [1]. It has several physical properties relevant to the photovoltaic solar energy conversion devices: quasidirect band light absorption mechanism, dependence of the bandgap width on the nanocrystal size, resistance to Stebler-Vronsky effect and possibility to be formed on flexible substrates. The use of the nanocomposite silicon as base material of the SC can substantially increase its efficiency by creating cascade type polymorphic heterostructures $[2,3]$ to reduce the cost of SC production due to advantages of thin film and roll technologies[4,5]. Among the main issues hampering the practical realization of nc-Si benefits, there is a lack of technology to control the size and concentration of $\mathrm{Si}$ nanocrystals at economically justified rates of film formation. Therefore, despite the large number of existing nc-Si manufacturing technologies, much attention is devoted to their improvement and search for the new ones [6-12].

One of promising ways in this direction is the use of metal-induced crystallization (MIC) of amorphous silicon [13-17]. In particular, the possibility to form $\mathrm{Si}$ 
nanocrystals in amorphous Si matrix at 2 to $5 \mathrm{~nm}$ sizes and the phase volume fraction up to $80 \%$ was shown by means of tin-stimulated crystallization of amorphous $\mathrm{Si}$ at low temperatures [18-20]. These experimental results interpreted by the new MIC mechanism proposed in the papers [20-22]. It differs significantly from those known for other metals [13, 15-17]. According to this mechanism, silicon nanocrystals are formed due to cyclic repetition of formation and decomposition of a supersaturated silicon in tin solution on the narrow eutectic layer at a-Si/Sn interface of tin micro-droplets in amorphous Si volume. The tin-induced crystallization of amorphous silicon can be stimulated by the action of laser light [23]. This allows us to measure the temperature, size and volume fraction of nanocrystals in the process of their formation by Raman scattering and simultaneously to control the crystallization process by changing the intensity and duration of laser radiation. The purpose of this work is to determine the temperature and time parameters of the MIC in the system a-Si-Sn, the evaluation of the role of photo-ionization in $\mathrm{Si}$ nanocrystals formation and the prospects for using pulsed laser radiation to control the nanocrystal size and concentration at tin-induced crystallization of amorphous silicon. To do this, we investigated the effect of pulse intensity in the ranges from $1.4 \cdot 10^{4}$ up to $2.18 \cdot 10^{8} \mathrm{~W} / \mathrm{cm}^{2}$ at duration $10 \mathrm{~ns}$ and $150 \mu \mathrm{m}$ (lasers with the light wavelength $535 \mathrm{~nm}$ and $1.07 \mu \mathrm{m}$ ) on the size and volume fraction of nanocrystals in nc-Si/a-Si composite film.

\section{Experimental}

Three-layer film structures, which cross section is schematically shown in Fig. 1, were investigated. They are made by successive deposition of silicon and tin thermally evaporated in vacuum on a monocrystalline silicon substrate at the temperature 150 to $200{ }^{\circ} \mathrm{C}$. All three deposition processes were carried out in a single vacuum chamber without depressurization at residual pressure $10^{-3} \mathrm{~Pa}$ by successive use of 3 different evaporators. Sprayed out Si (99.999\%) and Sn (99.92\%). The values of layer thickness in the sequence from substrate $\mathrm{X}, \mathrm{Y}, \mathrm{Z}$ are given in Table together with the parameters of laser irradiation.

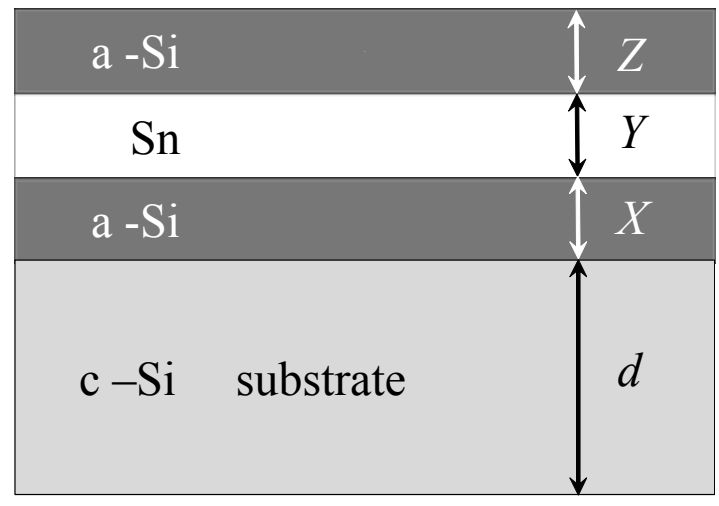

Fig. 1. Scheme of cross-section of layered $\mathrm{Si}-\mathrm{Sn}-\mathrm{Si}$ structures investigated, $d=1 \mathrm{~mm}$.

Table. Samples and modes of their laser processing.

\begin{tabular}{|l|l|l|l|l|}
\hline $\begin{array}{c}\text { Sample } \\
\text { No }\end{array}$ & $\begin{array}{c}\text { Thickness of the } \\
\text { layers X-Y-Z }(\mathrm{nm})\end{array}$ & \multicolumn{1}{|c|}{$\begin{array}{c}\text { Laser wavelength } \\
(\mathrm{nm})\end{array}$} & \multicolumn{1}{|c|}{ Pulse duration } & \multicolumn{1}{c|}{$\begin{array}{c}\text { Light power range } \\
\left(\mathrm{W} / \mathrm{cm}^{2}\right)\end{array}$} \\
\hline 1 & $50-100-200$ & 1070 & $150 \mu \mathrm{s}$ & $(1.4 \ldots 2.9) \cdot 10^{4}$ \\
\hline 2 & $\begin{array}{l}50-100-200 \\
50-100-200\end{array}$ & 1070 & $\begin{array}{l}150 \mu \mathrm{s} \\
10 \mathrm{~ns}\end{array}$ & $\begin{array}{l}1.3 \cdot 10^{5} \\
(5.3 \ldots 18.0) \cdot 10^{7}\end{array}$ \\
\hline 3 & $50-100-200$ & 1070 & $150 \mu \mathrm{s}$ & $(2.9 \ldots 7.8) \cdot 10^{4}$ \\
\hline 4 & $50-100-200$ & 535 & $10 \mathrm{~ns}$ & $(5.5 \ldots 8.5) \cdot 10^{6}$ \\
\hline 5 & $50-100-200$ & 535 & $10 \mathrm{~ns}$ & $(8.5 \ldots 21.75) \cdot 10^{6}$ \\
\hline 6 & $50-100-200$ & 1070 & $150 \mu \mathrm{s}$ & $(2.3 \ldots 6.9) \cdot 10^{4}$ \\
\hline $6-1$ & $50-100-200$ & 1070 & $10 \mathrm{~ns}$ & $(7.4 \ldots 15.3) \cdot 10^{7}$ \\
\hline $6-2$ & $50-100-200$ & 535 & $10 \mathrm{~ns}$ & $(3.5 \ldots 10.3) \cdot 10^{6}$ \\
\hline 7 & $0-100-200$ & 1070 & $10 \mathrm{~ns}$ & $(8.4 \ldots 52.0) \cdot 10^{7}$ \\
\hline 8 & $0-100-200$ & 1070 & $10 \mathrm{~ns}$ & $(2.0 \ldots 21.8) \cdot 10^{7}$ \\
\hline
\end{tabular}


The samples were marked by rectangular sections of $0.5 \times 0.5 \mathrm{~cm}$, each of which was irradiated by single pulses of a laser in a software scan mode with a controlled laser dot overlap at one power in one of the modes shown in Table. In our experiments, the diameter of the light beam spot was $70 \mu \mathrm{m}$, and the scan step was $50 \mu \mathrm{m}$. Therefore, we can assume that the entire surface of each site was exposed to the same irradiation. Different areas were irradiated by pulses of a varying power. Power regulation of laser light was carried out by a focusing system and attenuation of glass plates and neutral-gray filters. Thus, a series of plots was obtained for each sample, each of which was irradiated with different power at the same pulse duration and light wavelength. The phase composition of each site was then investigated by measuring and analyzing the Raman spectra. In addition, the image was taken using an optical microscope from the surface in the area around the place of measurement of the Raman spectrum.

Measurements of the micro-Raman spectra were carried out at room temperature in the inverse scattering geometry using a Horiba Jobin Yvon T64000 spectrometer equipped with the Olympus BX41 confocal microscope and a thermoelectric-cooled CCD detector. Excitation of the Raman spectra was performed using the Ar-Kr laser radiation with the wavelength $488 \mathrm{~nm}$. Spectral resolution was $\sim 0.15 \mathrm{~cm}^{-1}$. The excitation radiation was focused onto the surface of the test specimen with an Olympus $10 \times / 0.25$ lens in the area of $\sim 5 \mu \mathrm{m}^{2}$. Measurements were made at the power of laser radiation at the sample $I=1 \mathrm{~mW}$, which provided the power density close to $20 \mathrm{~kW} \cdot \mathrm{cm}^{-2}$. This irradiation did not lead to significant laser heating the samples as compared to their original room temperature.

\section{Results and discussions}

The typical Raman spectra within the range $100 \ldots 850 \mathrm{~cm}^{-1}$ of the original samples are shown in Fig. 2. The spectra of the samples Nos 7 and 8 prior to laser treatment contain only one wide band $(a)$ with a maximum near $475 \mathrm{~cm}^{-1}$ [24], which is characteristic of purely amorphous $\mathrm{Si}$. After certain modes of laser treatments, there additionally appears the narrow band with a maximum within the range $500 \ldots 520 \mathrm{~cm}^{-1}(b)$, which corresponds to the nanocrystalline phase of silicon [24, 25]. This is the result of a MIC of amorphous silicon under the influence of laser irradiation [23]. Initial spectra of the simples Nos 1 to 62 contain both bands.

That is, these samples contain both amorphous and crystalline phases even before laser treatments. They are used to study the effects of laser radiation on pre-formed nanocrystals. Determination of the crystal sizes $(L)$ and the fraction $\left(X_{C}\right)$ of volume occupied by crystals in the samples under investigation was carried out by using computer approximation of the Raman spectra as based on the theory of spatially bounded phonons [24, 25] with simplifications described in [20]. In particular, it was found that in the samples Nos 1, 2, 3, 6, 6-1 initial parameters of crystallinity make up: $L=$ $1.5 \mu \mathrm{m}, X_{C}=48 \%$. The change of these parameters under the influence of single laser pulses of different intensity, duration and wavelengths are considered below.

\section{Investigation of the effect of irradiation intensity $\lambda=1.07 \mu \mathrm{m}, \tau_{p}=150 \mu \mathrm{s}$}

The change of sizes and volume fraction of nanocrystals in the samples Nos 3 and 6 due to increasing the laser irradiation intensity after scanning by single pulses is shown in Fig. 3. Increasing the nanocrystal size and crystalline phase proportion is seen at enhanced power of laser light from approximately $5.5 \cdot 10^{4} \mathrm{~W} / \mathrm{cm}^{2}$. In particular, increasing the radiation power (from 5.5 to 7.8 ) $10^{4} \mathrm{~W} / \mathrm{cm}^{2}$ (i.e., by $42 \%$ ) leads to the nanocrystal size increase from 1.5 to $5.0 \mathrm{~nm}$, i.e., by $230 \%$. Likewise, the proportion of the crystalline phase volume changes similarly. A larger dispersion of the $X_{C}$ values (in comparison with distribution of nanocrystal sizes) is due to a greater statistical error in the calculation of this parameter. These results confirm the conclusions of recent work [23] about the irradiation intensity effect on the crystal size and concentration under MIC in Si$\mathrm{Sn}$-Si structures being influenced by continuous laser radiation. As can be seen from Fig. 3, this effect has a threshold in the region of $5 \cdot 10^{4} \mathrm{~W} / \mathrm{cm}^{2}$, which may be related, in particular, with achievement of the melting temperature for the tin layer in the investigated structures. According to $[21,22]$, the transition of tin into a liquid state is a prerequisite for MIC of amorphous Si.

Characteristically, with the increase of $I$ from $5 \cdot 10^{4}$ up to $8 \cdot 10^{4} \mathrm{~W} / \mathrm{cm}^{2}$ (and, accordingly, the temperature in the laser beam spot), the growth of the crystalline phase part of volume is much slower than the nanocrystal size growth, although the crystal volume $\sim L^{3}$. This means that only part of the output nanocrystals serves as the nuclei for precipitation of Si solution in Sn. The majority of them do not grow, because they have a size smaller than the nucleus critical one. Fig. 4 shows the optical microscope images of samples surface output and irradiated by subthreshold in capacities range $(a)$, as well as of the area irradiated by pulses of the maximum power $(b)$. Dark spots correspond to regions with a high concentration of the crystalline phase.

It is seen that such areas are already observed in the initial sample. They remain irreplaceable after laser irradiation at the intensity down threshold. There is an increase in the size of these areas at the intensity over threshold. The increase in the total area of dark areas correlates with the increase of $X_{C}$. 
Semiconductor Physics, Quantum Electronics \& Optoelectronics, 2017. V. 20, N 4. P. 396-405.

doi: https://doi.org/10.15407/spqeo20.04.396

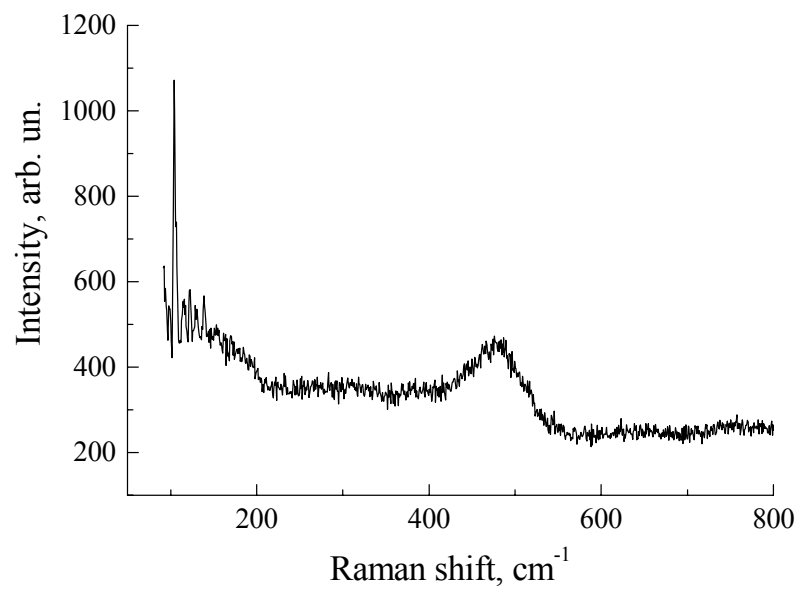

a)

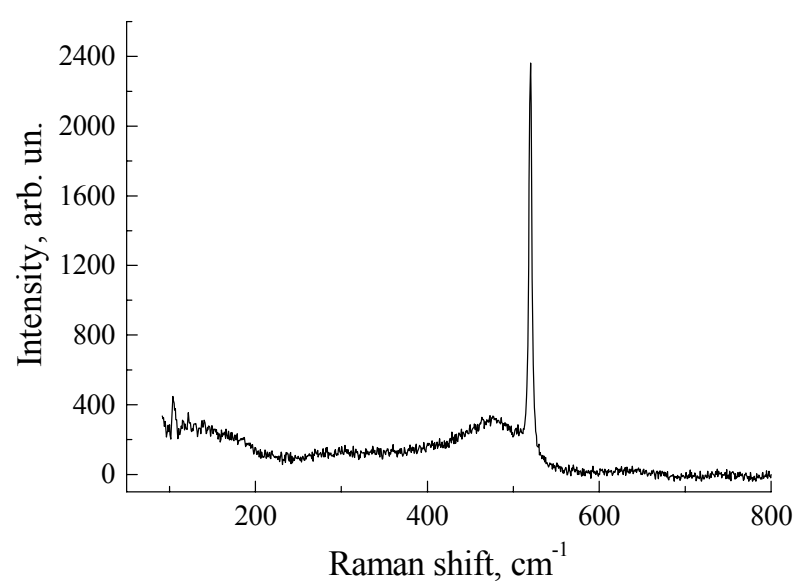

b)

Fig. 2. Typical Raman spectra for amorphous (a) and partially crystallized (b) silicon.

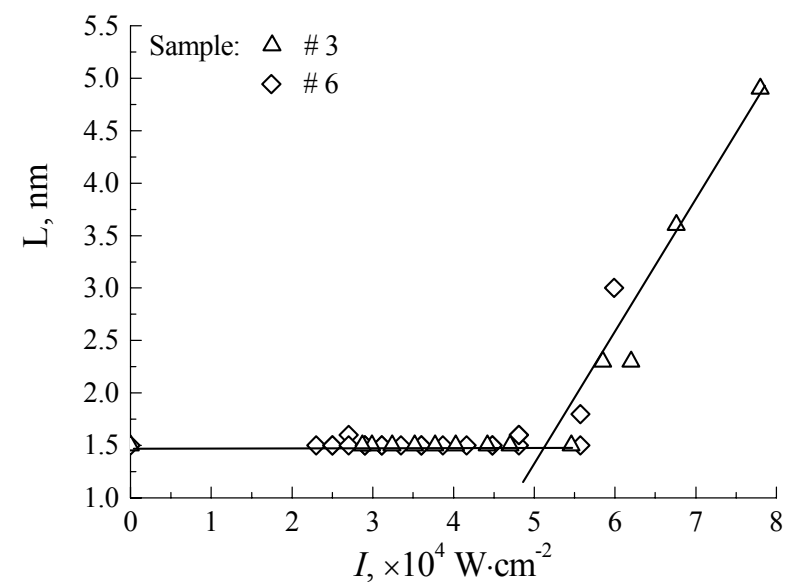

a)

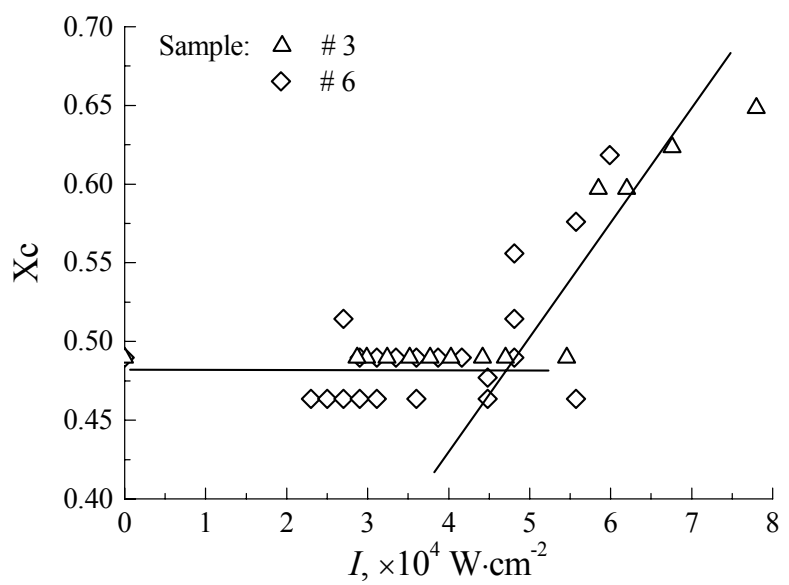

b)

Fig. 3. Dependence of the nanocrystal size $(a)$ and volume fraction $(b)$ on the laser irradiation power for the samples No. 3 and No. 6. The points indicate experimental data, lines are linear approximation.
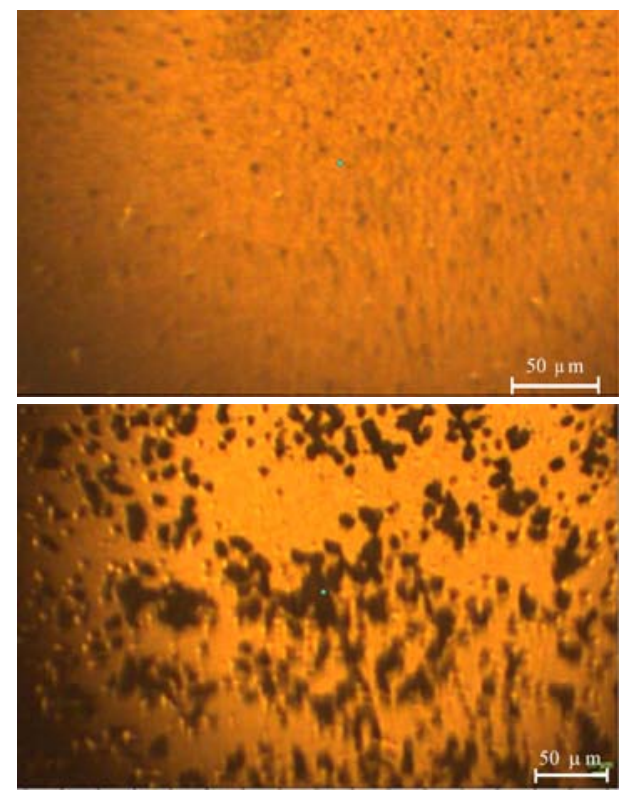

Fig. 4. Optical microscope images of sample surface after laser irradiation with the power: a) $I=(0 \ldots .5) \cdot 10^{4} \mathrm{~W} / \mathrm{cm}^{2}$; b) $I=$ $8.2 \cdot 10^{4} \mathrm{~W} / \mathrm{cm}^{2}$.

\section{Investigation of radiation exposure in the mode $\lambda=1.07 \mu \mathrm{m}, \tau=10 \mathrm{~ns}$}

Similar to previous case, samples Nos 2 and 6-1 (3-layer structure Si-Sn-Si (50-100-200 nm) partially crystallized $\left(L=1.5 \mathrm{~nm}, X_{C}=48 \%\right)$ were irradiated by light with the same $\lambda=1070 \mathrm{~nm}$, but four orders of magnitude shorter $(10 \mathrm{~ns})$ and three orders of magnitude more powerful pulses $\left(\sim 10^{7} \mathrm{~W} / \mathrm{cm}^{2}\right)$. The results of the influence of above irradiation on the crystallinity parameters of silicon are shown in Fig. 5.

As in the previous case, it is seen that there is a threshold (here in the region of approximately $7.5 \cdot 10^{7} \mathrm{~W} / \mathrm{cm}^{2}$ ) of the crystallization dependence on the of laser irradiation intensity. As in the preceding case, a microscope shows correlation of laser pulse intensity and growth of the total area of dark spots due crystallization regions at super-threshold powers. Knowing the power and duration of the laser pulses in both cases, it is easy to estimate and compare the density of the energy absorbed at crystallization threshold: $E^{t c}=I \tau$. It turned out that in the first case $E_{1}{ }^{t c}=$ $8.3 \mathrm{~J} / \mathrm{cm}^{2}$, and in the second one $E_{2}{ }^{t c}=0.75 \mathrm{~J} / \mathrm{cm}^{2}$. That

(C) 2017, V. Lashkaryov Institute of Semiconductor Physics, National Academy of Sciences of Ukraine 
is, in the case of more powerful laser irradiation, the beginning of the MIC requires an order of magnitude less energy. This can be explained in at least two ways: stimulating influence of photoionization [23, 26] or greater heating by a nanosecond pulse as compared with microsecond pulse in the consequence of heat transfer inertia.

Fig. 6 shows the experimental dependences of crystal size and volume fraction of the crystalline phase on the $10 \mathrm{~ns}$ pulse power in the sample No. 7. Its initial state was completely amorphous. The pulse power up to $1.5 \cdot 10^{8} \mathrm{~W} / \mathrm{cm}^{2}$ does not cause phase transformations in an amorphous film. At higher densities, modification of the film occurs, and the crystalline component appears. Moreover, the size of the crystals immediately exceeds $10 \mathrm{~nm}$. At the pulse power $2.0 \cdot 10^{8} \mathrm{~W} / \mathrm{cm}^{2}$ and higher, only spectra of monocrystalline silicon substrates are recorded. This indicates that in the intensity range $I=$ $(1.5 \ldots 2.0) \cdot 10^{8} \mathrm{~W} / \mathrm{cm}^{2}$ an amorphous film is destroyed without significant crystallization. Qualitatively similar behavior is demonstrated by the sample No. 8 that also did not contain initial crystalline nuclei.

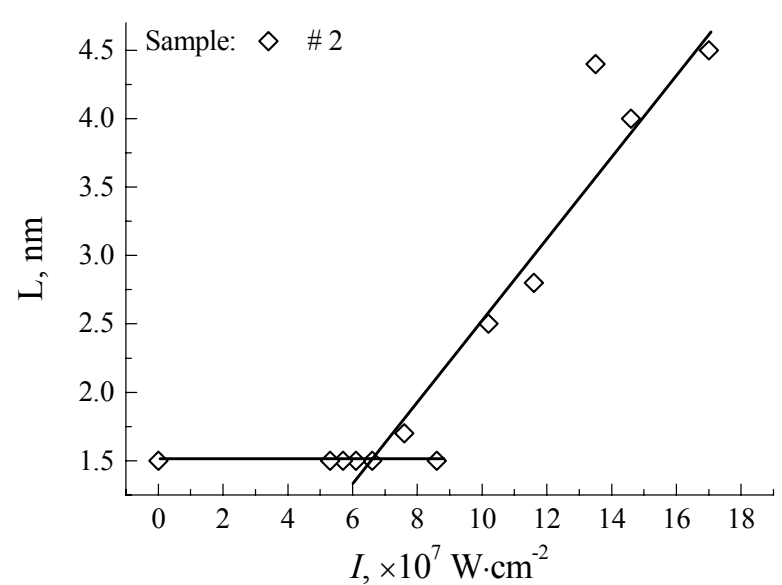

a)
Thus, in contrast to the above-mentioned amorphous-crystalline samples Nos 2 and 6, in whichthe growth of the crystalline phase with growth of the pulse power was clearly observed, pure amorphous samples Nos 7 and 8 do not demonstrate any influence of laser treatment on MIC. Therefore, it can be argued that the MIC process involves formation of nuclei (incubation period) for more than $10 \mathrm{~ns}$ and a phase of their rapid growth (from 1.5 up to $4.5 \mathrm{~nm}$ ) for times approximately $10 \mathrm{~ns}$.

\section{Investigation of the laser pulse $\left(\lambda=535 \mathrm{~nm}, t_{p}=10 \mathrm{~ns}\right)$ intensity influence on MIC}

In contrast to light with $\lambda=1070 \mathrm{~nm}$, which is weakly absorbed in a-Si and heats mainly the tin layer in the investigated structures, the light with $\lambda=535 \mathrm{~nm}$ is almost completely absorbed in the outer layer of amorphous silicon. The samples Nos 4, 5, and 6-2 were irradiated with laser light of the wavelength $535 \mathrm{~nm}$, pulse duration of $10 \mathrm{~ns}$, as in the previous case with $\lambda=$ $1070 \mathrm{~nm}$, in the power range $\sim 10^{6} \ldots 10^{7} \mathrm{~W} / \mathrm{cm}^{2}$. The results of measurements and analysis of the Raman spectra are shown in Fig. 7.

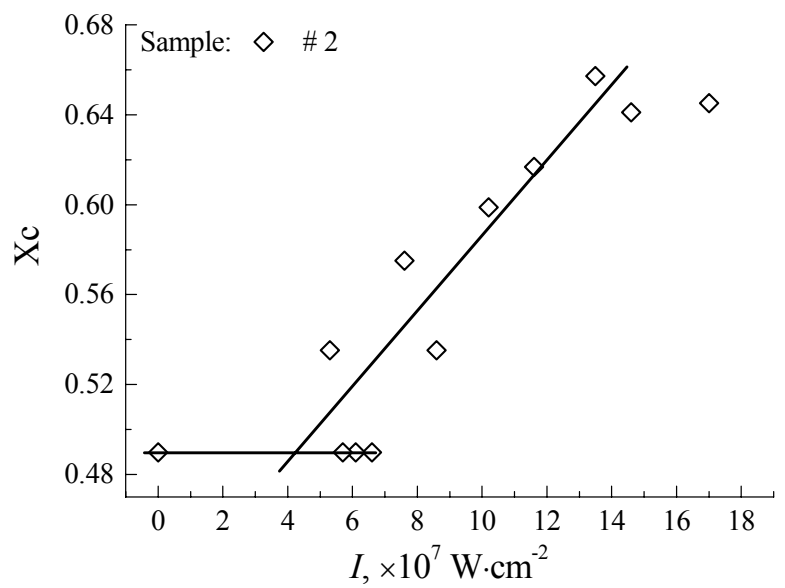

b)

Fig. 5. Crystals size $L$ dependence ( $a$ ) and crystal phase volume fraction $X_{C}(b)$ in the outer layer of the Si sample No. 2. Points correspond to experimental data, lines are linear interpolation.

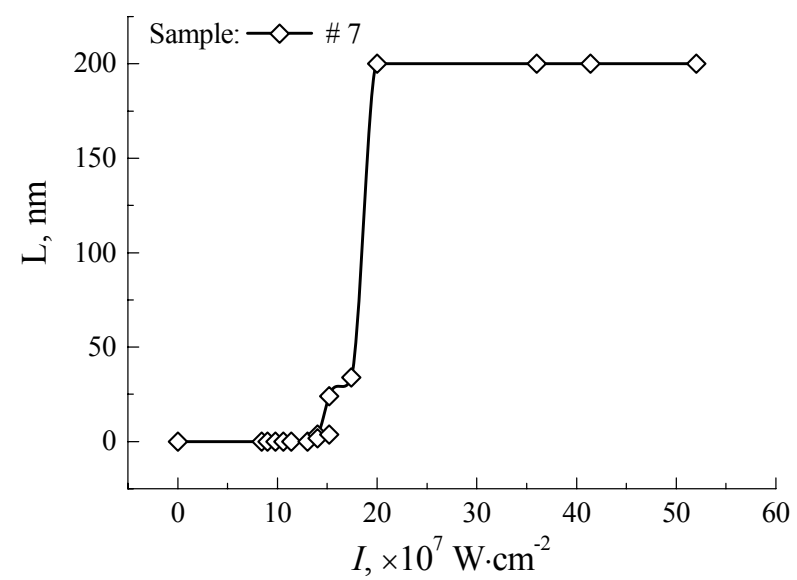

a)

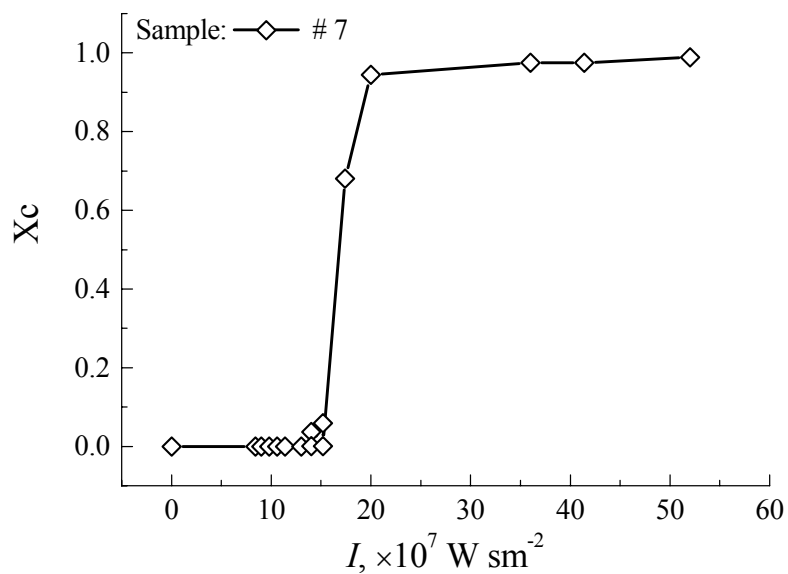

b)

Fig. 6. Dependence of the size $(a)$ and volume fraction $(b)$ of Si crystals on the laser pulse $(10 \mathrm{~ns})$ power in the sample No. 7. 


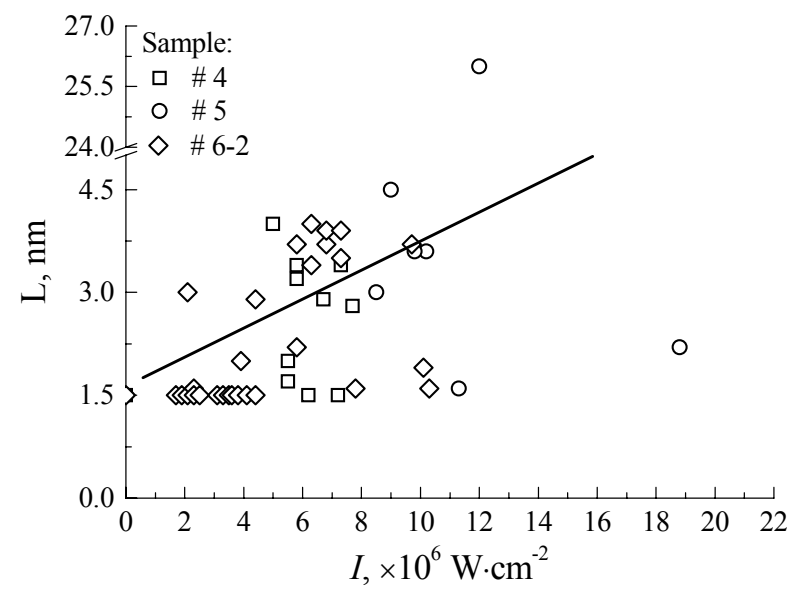

a)

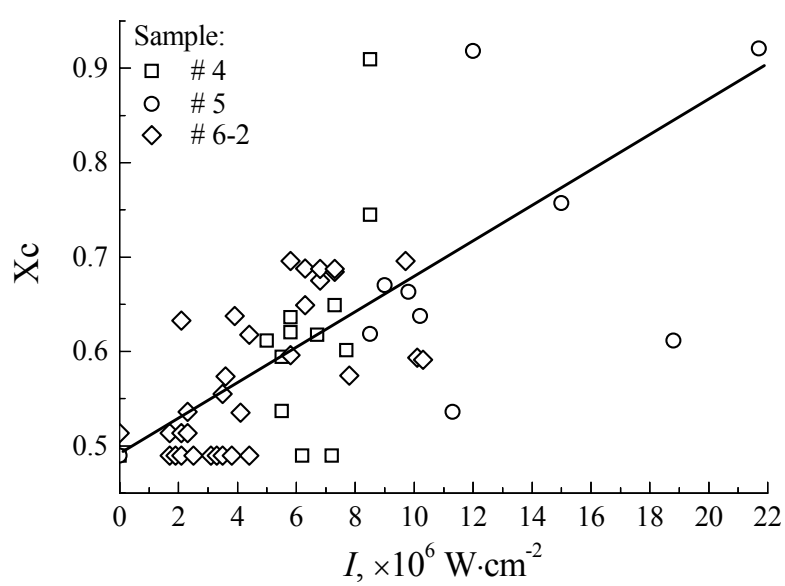

b)

Fig. 7. Dependence of the size (a) and volume fraction of Si nanocrystals on the laser pulse power for the samples Nos 4, 5, 6-2.

As one can see, there is much more bigger spreading of crystallinity parameters values than in previous studies. This may be caused by the uneven phase transformation of outer a-Si film. It should be noted that each point in the plot corresponds to different sections of surface treated by laser beam with a spot diameter of $70 \mu \mathrm{m}$ with the scan step $50 \mu \mathrm{m}$. At the same time, the diameter of light beam used for Raman measurements is $\sim 2 \mu \mathrm{m}$. When treating with longwave light $(1070 \mathrm{~nm})$ that is absorbed mainly in the heat conducting metal layer of the investigated structures, the possible heterogeneities in heating may be equalized during the laser pulse. In contrast to treatment by more short-time and short-wave $(535 \mathrm{~nm})$ light that is strongly absorbed by weakly thermal conductive layer of amorphous silicon. Indeed, the thermal depth is determined from the ratio [27]:

$l_{t h}=\left[(æ+D) \cdot t_{p}\right]^{1 / 2}$

(where $\mathfrak{x}$ is the thermal diffusivity; $D$ - coefficient of bipolar diffusion of non-equilibrium charge carriers (in semiconductors); $t_{p}$-duration of laser pulse).

For tin (temperature conductivity $\mathfrak{x}=0.37 \mathrm{~cm}^{2} / \mathrm{s}$, $\left.t_{p}=10^{-8} \mathrm{~s}\right) l_{t h}=6.1 \cdot 10^{-5} \mathrm{~cm}$.

At the same time, the absorption coefficient of green light for amorphous silicon is $\alpha=6 \cdot 10^{4} \mathrm{~cm}^{-1}$, absorption depth $l_{\alpha}=1 / \alpha=1.6 \cdot 10^{-5} \mathrm{~cm}$, and the heat depth $l_{t h}=8 \cdot 10^{-6} \mathrm{~cm}$. (In this case, the depth of warming is determined by the higher value, that is, the depth of absorption $l_{\alpha}=1 / \alpha$.)

But in the lateral dimension (in area) the thermal depth dominates. This is an order of magnitude less than the thickness of outer layer of amorphous silicon in the investigated structures. As a result of worst heat conditions, the maximum local heating temperature and, therefore, the temperature gradient increases. This can cause large local deformation stresses and, consequently, greater damage in the outer layer of a-Si. Even though the short-wave irradiation power of 10-ns pulses is an order of magnitude lower than that of the longwave one.

Indeed, shown in Fig. 8 are the microphotographs of surface of the samples under investigation before and after laser irradiation. It is seen that this type of laser irradiation leads to a fundamental change in the surface state as compared to the original one.

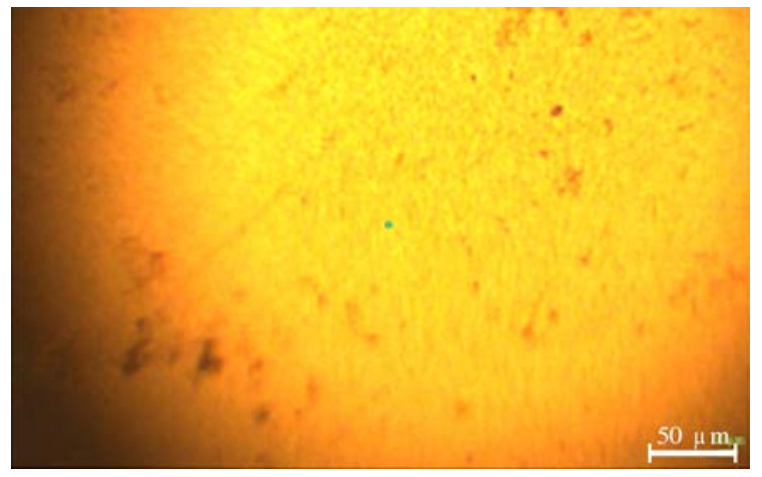

a)

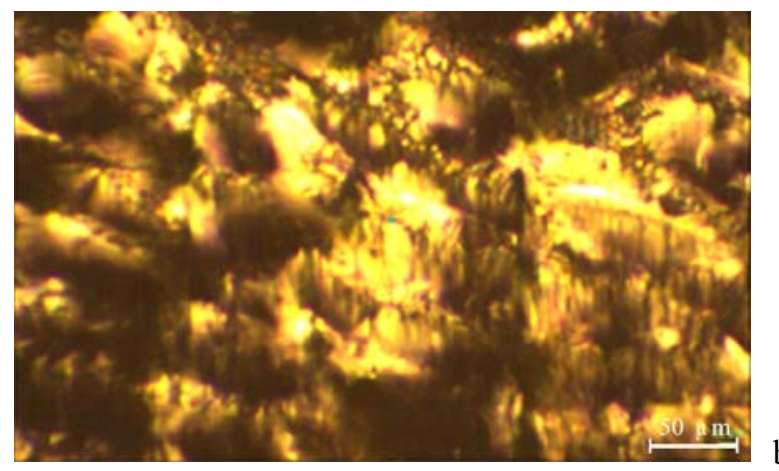

b)

Fig. 8. Influence of the laser $(535 \mathrm{~nm}, 10 \mathrm{~ns})$ intensity on the surface of a-Si as seen in an optical microscope: a) $I=0$; b) $I=$ $(2 \ldots 20) \cdot 10^{6} \mathrm{~W} / \mathrm{cm}^{2}$. 
To estimate the temperature conditions for MIC under pulsed laser irradiation, we considered formation of an laser induced non-equilibrium temperature distribution in one-dimensional approximation (along the thickness of the investigated structures). To do this, we use the one-dimensional heat equation based on the Fourier law:

$c \rho \frac{\partial \theta}{\partial t}=\left(\frac{\partial}{\partial z} K \frac{\partial \theta}{\partial z}\right)+P(z, t)$,

where $c$ and $\rho$ are the specific heat density and density of the investigated medium, respectively, $K$ is the thermal conductivity coefficient, $P(z, t) \quad-$ function that characterizes the spatial and temporal distribution of bulk heat sources.

In a linear approximation, one can separate the spatial and temporal components of the distribution:

$P(z, t)=f(z) g(t)$.

In the case under consideration, the spatial distribution of bulk heat sources can be represented as follows:

$$
f(z)=I_{0} \alpha(z) \exp \left(-\int_{0}^{z} \alpha\left(z^{\prime}\right) d z^{\prime}\right),
$$

where $\alpha(z)$ is the spatial distribution of the optical absorption coefficient.

In all cases, as the initial condition, the condition for the absence of temperature rise in the investigated structure was used $(\theta(z, 0)=0)$.

Let's analyze the laser heating caused by pulse duration of $150 \mu \mathrm{s}$. In this case, the length of laser pulse fronts are much less than its total duration. Therefore, the pulse shape was considered to be rectangular:

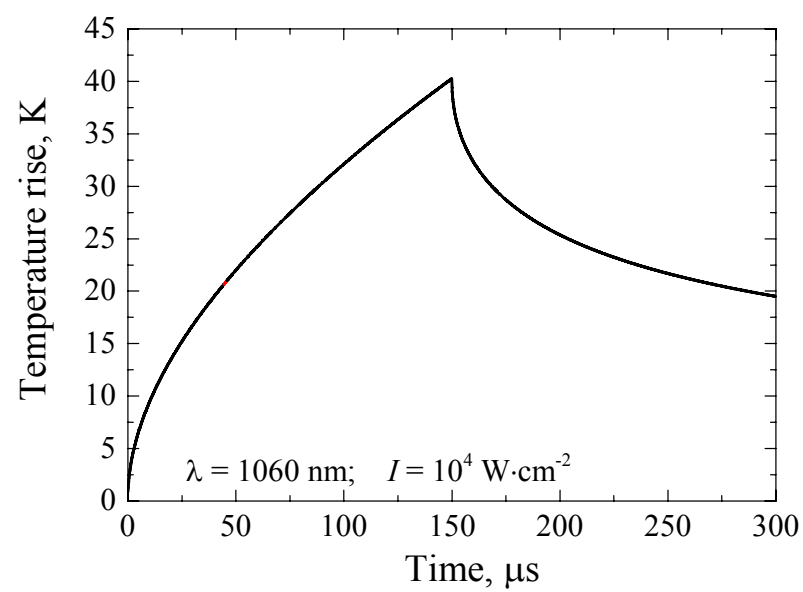

a) $g(t)= \begin{cases}1, & t \leq \tau_{p} \\ 0, & t>\tau_{p}\end{cases}$

where $\tau_{p}=150 \mu$ s pulse duration.

Since the pulse duration is much more bigger than typical time of heat propagation in deposited layers, the approach of heat propagation in the crystal substrate was considered. In this case, volume heat sources in the amorphous layer can be considered as heat sources arising in the upper boundary of the sample:

$\left.K \frac{\partial \theta}{\partial z}\right|_{z=0}=P_{S}(t)$,

where

$P_{S}(t)=\int_{0}^{X+Y+Z} P(z, t) d z$.

In addition, the boundary condition of absence of heat outflow from back surface of sample was used:

$\left.K \frac{\partial \theta}{\partial z}\right|_{z=X+Y+X+D}=0$.

The resulting temporal dependence of temperature on sample surface at laser radiation power equal to $10^{4} \mathrm{~W} / \mathrm{cm}^{2}$ is presented in Fig. 9a. A typical dependence of the spatial temperature distribution in the investigated structure is presented in Fig. $9 \mathrm{~b}$ at the time $t=\tau_{p}$.

According to Fig. 5, the threshold of structural state change occurs in the region of intensity near $10^{4} \mathrm{~W} / \mathrm{cm}^{2}$, that is, at the intensity 5 times lower than that used in

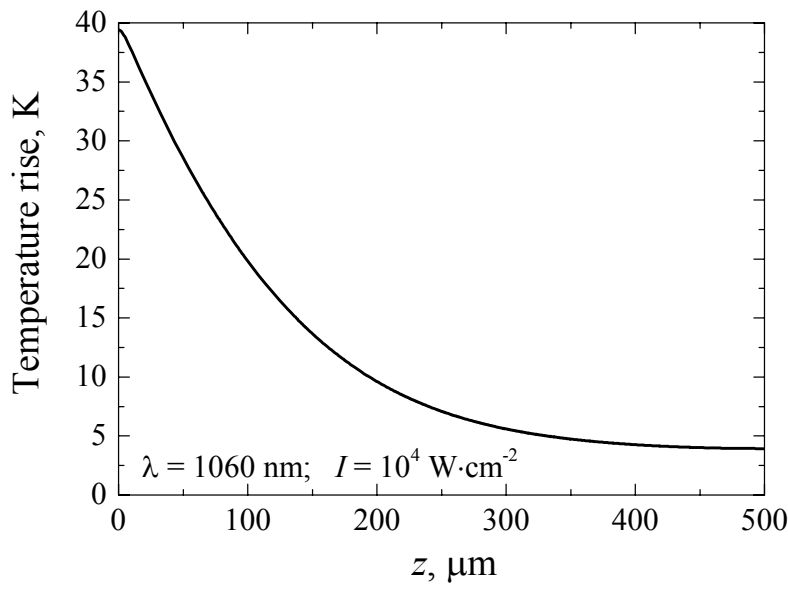

b)

Fig. 9. Estimated temperature growth on the irradiated sample surface during the laser pulse and immediately after it (a); temperature distribution over the thickness of the sample after laser pulse having finished $(b)$. 
calculation, the results of which are shown in Figs. 9a and $9 \mathrm{~b}$. Therefore, to estimate the temperature at the intensity $5 \cdot 10^{4} \mathrm{~W} / \mathrm{cm}^{2}$ in linear approximation, we multiply the temperature obtained at the intensity $10^{4} \mathrm{~W} / \mathrm{cm}^{2}$ by 5 and add room temperature $(300 \mathrm{~K})$. This corresponds to the maximum temperature on surface: $300 \mathrm{~K}+40 \cdot 5=500 \mathrm{~K}$. This temperature is close to $T$ of tin melting $\left(230{ }^{\circ} \mathrm{C}+273 \mathrm{~K}=503 \mathrm{~K}\right)$. At the maximum power near $8 \cdot 10^{4} \mathrm{~W} / \mathrm{cm}^{2}$ (Fig. 9), the estimated temperature will be $300+40 \cdot 8=620 \mathrm{~K} \sim 350{ }^{\circ} \mathrm{C}$, which is consistent with the data of previous studies on the tin induced crystallization of amorphous silicon [20, 21].

In the case of pulses of nanosecond duration, the spatial distribution of thermophysical and optical parameters in the irradiated structure plays a significant role. Therefore, in this case, these spatial distributions were considered in more detail. In particular, Fig. 10 shows the spatial distribution of heat sources $(f(z))$ and the time distribution of temperature on the structure surface for two wavelengths (532 and $1064 \mathrm{~nm}$ ) at laser pulse power $1 \mathrm{~W} / \mathrm{cm}^{2}$.

In this case, the time distribution of the intensity in a laser pulse was considered as obeying the Gaussian shape:

$$
g(t)=\exp \left(-4 \log (2) \frac{\left(t-\tau_{p}\right)^{2}}{\tau_{p}^{2}}\right)
$$

As can be seen from Fig. 10a, in the case of irradiation with infra-red radiation, heating the medium is mainly associated with absorption of radiation in the tin layer.

As shown in Fig. $10 \mathrm{~b}$ at such laser power, the temperature on the structure surface is close to the melting point of tin in the case of irradiation with the wavelength $\lambda=532 \mathrm{~nm}$. It correlates with the data presented in Fig. 7 - crystallization begins already at a minimum intensity of laser pulse. The intensity of $1 \mathrm{~W} / \mathrm{cm}^{2}$ is not sufficient to start crystallization in the case of light irradiation with the wavelength $\lambda=$ $1064 \mathrm{~nm}$. However, at the intensity of $60 \mathrm{~W} / \mathrm{cm}^{2}$ when the crystallization threshold is observed experimentally, in accordance with the calculations, the temperature in the surface layer should reach, in a linear approximation, $300 \mathrm{~K}+30 \cdot 60=2100 \mathrm{~K}$, which significantly exceeds the melting point of tin. This is non-coincidence of experimental data and model calculations may be indicative of nonlinear optics phenomena, in particular, the effect of absorption saturation [28]. We can assume that the significantly reduced density of electronic states is in nano-fragments as compared with that in volume. Therefore, optical absorption and corresponding heating will have substantially lower values. The nanostructures of thin tin layers in similar structures of Si-Sn-Si were observed in [20] at manufacture conditions like those used here.

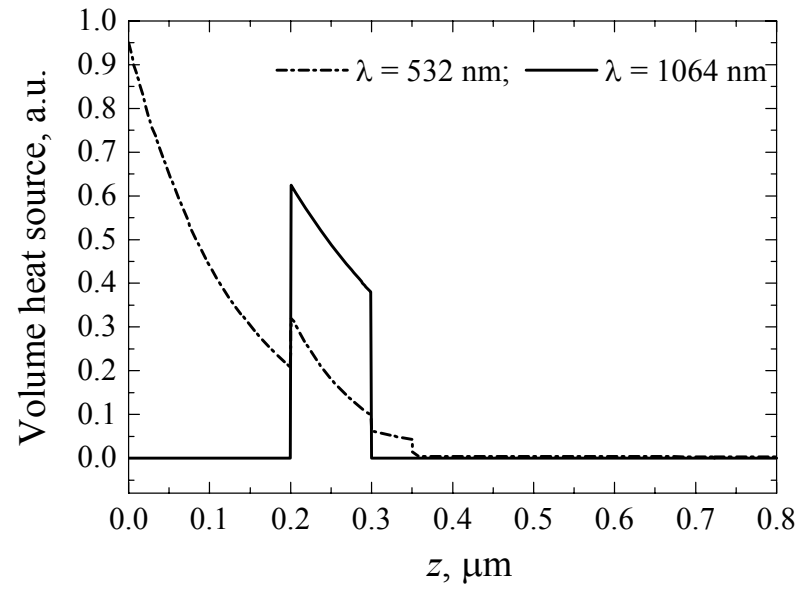

a)

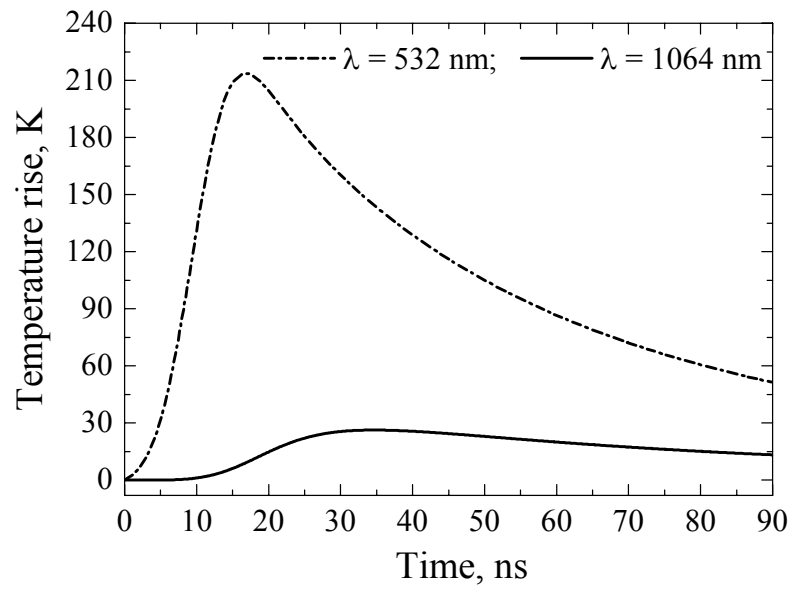

b)

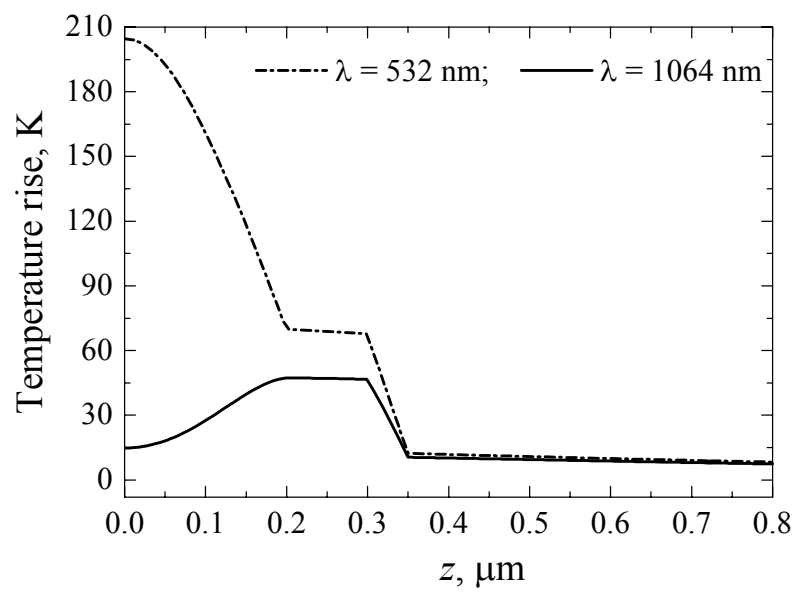

c)

Fig. 10. Estimated distribution of heat sources $(f(z))$ in the thickness of Si-Sn-Si structure $(a)$; heating the structure surface with time $(b)$; temperature fields at the time $t=2 \tau_{p}(c)$ at irradiation by 10 -ns pulses of infrared and green lasers with the power $1 \mathrm{~W} / \mathrm{cm}^{2}$. 


\section{Conclusions}

The coincidence in temperatures for the beginning of structural-phase changes and tin melting confirms the mechanism of tin-induced crystallization of amorphous silicon as a process of cyclic formation and decaying of a liquid solution of silicon in tin, proposed in papers $[20,21]$.

The discovered here two-stage MIC - the presence a long incubation period of nuclei formation and a rapid phase of their growth - is characteristic for processes of solutions decay, which may also be an argument in favor of the indicated mechanism.

The obtained results indicate the possible influence of photoionization on MIC of amorphous silicon.

Due to high speed of crystallization of amorphous silicon, induced by tin $\left(10^{-8} \ldots 10^{-4} \mathrm{~s}\right)$, its stimulation by pulsed light irradiation can be the basis for new technologies of controlling the nanocrystal size in the nc-Si film production.

\section{References}

1. Beard M.C., Luther J.M., and Nozik A.J. The promise and challenge of nanostructured solar cells. Nature nanotechnology. 2014. 9, No. 12. P. 951-954.

2. Alferov Z.I., Andreev V.M., and Rumyantsev V.D. Solar photovoltaics: Trends and prospects. Semiconductors. 2004. 38, Issue 8. P. 899-908.

3. Yan B., Yue G., Xu X., Yang J., and Guha S. High efficiency amorphous and nanocrystalline silicon solar cells. phys. status solidi. 2010. 207, Issue 3. P. 671-677.

4. Lewis N.S. Toward cost-effective solar energy use science. Science. 2007. 315, Issue 5813. P. 798801.

5. Sondergaard R., Hosel M., Angmo D., LarsenOlsen T.T., and Krebs F.C. Roll-to-roll fabrication of polymer solar cells. Mater. Today. 2012. 15, No. 1. 36-49.

6. Birkholz M., Selle B., Conrad E., Lips K., and Fuhs W. Evolution of structure in thin microcrystalline silicon films grown by electron-cyclotron resonance chemical vapor deposition. J. Appl. Phys. 2000. 88, No. 7. P. 4376-4379.

7. Rech B., Roschek T., Müller J., Wieder S., and Wagner H. Amorphous and microcrystalline silicon solar cells prepared at high deposition rates using RF (13.56 MHz) plasma excitation frequencies. Sol. Energy Mater. Sol. Cells. 2001. 66, No. 1. 267-273.

8. van Veen M.K., van der Werf C.H.M., and Schropp R.E.I. Tandem solar cells deposited using hot-wire chemical vapor deposition. J. Non.-cryst. Solids. 2004. 338-340. P. 655-658.

9. Mai Y., Klein S., Carius R., Stiebig H., Houben L., Geng X., and Finger F. Improvement of open circuit voltage in microcrystalline silicon solar cells using hot wire buffer layers. J. Non.-cryst. Solids. 2006. 352. P. 1859-1831.

10. Li H., Franken R.H., Stolk R.L., van der Werf C.H.M., Rath J.K., and Schropp R.E.I. Controlling the quality of nanocrystalline silicon made by hotwire chemical vapor deposition by using a reverse H2 profiling technique. J. Non.-cryst. Solids. 2008. 354, No. 19. P. 2087-2091.

11. Amrani R., Pichot F., Chahed L., and Cuminal Y. Amorphous-nanocrystalline transition in silicon thin films obtained by argon diluted silane PECVD. Cryst. Struct. Theory Appl. 2012. 1, No. 3. P. 57-61.

12. Fugallo G. and Mattoni A. Thermally induced recrystallization of textured hydrogenated nanocrystalline silicon. Phys. Rev. B. 2014. 89, No. 4. P. 045301.

13. Nast O. and Hartmann A.J. Influence of interface and $\mathrm{Al}$ structure on layer exchange during aluminum-induced crystallization of amorphous silicon. J. Appl. Phys. 2000. 88. P. 716.

14. Jeon M., Jeong C., and Kamisako K. Tin induced crystallisation of hydrogenated amorphous silicon thin films. Mater. Sci. Technol. 2010. 26, No. 7. P. 875-878.

15. Mohiddon M.A. and Krishna M.G. Growth and optical properties of $\mathrm{Sn}-\mathrm{Si}$ nanocomposite thin films. J. Mater. Sci. 2012. 47, No. 19. 6972-6978.

16. van Gestel D., Gordon I., and Poortmans J. Aluminum-induced crystallization for thin-film polycrystalline silicon solar cells: Achievements and perspective. Sol. Energy Mater. Sol. Cells. 2013. 119. P. 261-270.

17. Mohiddon M.A. and Krishna M.G. Chapter 17: Metal Induced Crystallization. In: Crystallization Science and Technology. Ed. By Marcello Rubens Barsi Andreeta. InTech, 2012.

18. Voitovych V.V., Neimash V.B., Krasko N.N., Kolosiuk A.G., Povarchuk V.Y., Rudenko R.M., Makara V.A., Petrunya R.V., Juhimchuk V.O., and Strelchuk V.V. The effect of Sn impurity on the optical and structural properties of thin silicon films. Semiconductors. 2011. 45, No. 10. Article 1281.

19. Neimash V.B., Poroshin V.M., Kabaldin A.M., Yukhymchuk V.O., Shepelyavyi P.E., Makara V.A., and Larkin S.Y. Microstructure of thin $\mathrm{Si}-\mathrm{Sn}$ composite films. Ukr. J. Phys. 2013. 58. P. 865.

20. Neimash V., Poroshin V., Shepeliavyi P., Yukhymchuk V., Melnyk V., Kuzmich A., Makara V., and Goushcha A.O. Tin induced a-Si crystallization in thin films of Si-Sn alloys. J. Appl. Phys. 2013. 114, No. 21. P. 213104.

21. Neimash V.B., Goushcha A.O., Shepeliavyi P.Y., Yukhymchuk V.O., Danko V.A., Melnyk V.V. and Kuzmich A.G. Mechanism of tin-induced crystallization in amorphous silicon. Ukr. J. Phys. 2014. 59. P. 1168-1176. 
22. Neimash V.B., Goushcha A.O., Shepeliavyi P.Y., Yukhymchuk V.O., Danko V.A., Melnyk V.V. and Kuzmich A.G. Self-sustained cyclic tin induced crystallization of amorphous silicon. J. Mat. Res. 2015. 30, No. 20. 3116-3124.

23. Neimash V., Shepelyavyi P., Dovbeshko G., Goushcha A.O., Isaiev M., Melnyk V., Didukh O., and Kuzmich A. Nanocrystals growth control during laser annealing of $\mathrm{Sn}:(\alpha-\mathrm{Si})$ composites. $J$. Nanomater. 2016. 2016. Article ID 7920238, 8 p.

24. Richter H., Wang Z.P., and Ley L. The one phonon Raman spectrum in microcrystalline silicon. Solid State Commun. 1981. 39, Issue 5. P. 625-629.

25. Campbell I.H. and Fauchet P.M. The effects of microcrystal size and shape on the one phonon
Raman spectra of crystalline semiconductors. Solid State Commun. 1986. 58, No. 10. P. 739-741.

26. Hiraki A. Low Temperature Reactions at $\mathrm{Si} /$ metal Interfaces; What Is Going on at the Interfaces? Surf. Sci. Repts. 1983. 3, Issue 7. P. 357-412.

27. Akhmanov S.A., Emel'yanov V.I., Koroteev N.I., Seminogov V.N., Interaction of powerful laser radiation with the surfaces of semiconductors and metals: nonlinear optical effects and nonlinear optical diagnostics. Sov. Phys. Uspekhi. 1985. 28, No. 12. P. 1084-1124.

28. Plaksin O., Takeda Y., Amekura H., Kishimoto N., and Plaksin S. Saturation of nonlinear optical absorption of metal-nanoparticle composites. $J$. Appl. Phys. 2008. 103. P. 114302. 